III. Inklusion über die Lebensspanne 
1. Alter und Behinderung 


\title{
Behinderung und Pflegebedürftigkeit im Alter - sind die sozialrechtlichen Reaktionen konsistent?
}

\author{
Gerhard Igl
}

I. Zusammenhänge und Zugänge zum Thema:

Behinderung, Pflegebedürftigkeit, Alter

II. Unterschiedliche Reaktionen des Sozialrechts auf die Zusammenhänge von „Alter und Behinderung“ und „Behinderung und Alter" mit Blick auf die Sicherung bei Pflegebedürftigkeit?

1. Unterschiedliche Lebenssituationen: Der alte Mensch mit Behinderung Der behinderte Mensch im Alter

2. Unterschiedliche sozialrechtliche Reaktionen auf diese unterschiedlichen Lebenssituationen?
a) Fragestellungen
b) Nichtökonomische Bedarfe im Alter und bei Behinderung
c) Ökonomischer Bedarf bei Alter und Behinderung
d) Krankheit im Kontext von Alter und Behinderung
e) Pflegebedürftigkeit im Kontext von Alter und Behinderung
f) Behinderung im Alter: Teilhabe und Rehabilitation
g) Die Wirkungen der Wahrnehmung von Alter, Behinderung und Pflegebedürftigkeit in den einzelnen Sozialleistungssystemen

III. Sozialleistungspraxis bei älteren behinderten Menschen

\section{Fragestellungen}

2. Allgemeine Probleme der Feststellung einer altersspezifischen Leistungspraxis bei Sozialleistungen

3. Zur Problematik der Leistungspraxis der Eingliederungshilfe für ältere behinderte Menschen

a) Fragestellungen

b) Versuch einer Annäherung an die Problematik

IV. Ergebnisse und Schlussfolgerungen

1. Sozialleistungsrecht

2. Sozialleistungsrechtliche Praxis

3 . Forschungsbedarf

4. Zur Eingangsfrage 


\section{Zusammenhänge und Zugänge zum Thema:}

\section{Behinderung, Pflegebedürftigkeit, Alter}

Das Thema Behinderung und Pflegebedürftigkeit im Alter und die Frage nach den sozialrechtlichen Reaktionen darauf ist als explizit so formuliertes Thema noch recht jung. ${ }^{1}$ Als implizit formuliertes Thema kursiert es in der sozialpolitischen Diskussion schon seit den sechziger Jahren. Man erinnere sich nur an die Diskussion um die Sicherung im Pflegefall, die sich von Beginn an sehr oft beschränkte auf eine Diskussion um den Schutz pflegebedürftiger älterer Menschen. ${ }^{2}$ Und mit der Bezugnahme auf diese Diskussion sind wir bei einer zentralen Fragestellung unseres Themas, die da lautet, ob sich unsere sozialrechtlichen Sichtweisen auf das Problem Alter und Behinderung oder Behinderung und Alter nicht zu sehr einengen auf den sozialrechtlichen Schutz bei Pflegebedürftigkeit, all dies in dem Wissen, dass die Leistungen bei Pflegebedürftigkeit vor allem von älteren Menschen in Anspruch genommen werden. ${ }^{3}$

Dass das Alter mit Behinderung konnotiert wird, ist üblich. Dass die Behinderung mit dem Alter in Verbindung gebracht wird, erscheint nicht so üblich. Es ist ein Unterschied zu sagen, ich bin ein alter Mensch und im Alter ein behinderter Mensch geworden, oder ich bin als behinderter Mensch alt geworden. Alter und Behinderung wird verstanden als „Alter mit Behinderung"; Behinderung und Alter wird verstanden als „Der behinderte Mensch im Alter“.

Und damit ist man bei einem ersten Unterschied, der sich dann auch sozialrechtlich niederschlagen kann: Alter und Behinderung legen eher nahe, dass die Behinderung altersbedingt ist. Pflegebedürftigkeit im Alter ist eine Form der Behinderung im Alter. Behinderung und Alter hingegen meint, dass die Behinderung schon vor dem Übergang in die Altersphase gegeben war.

Im Folgenden soll untersucht werden, wie das Sozialrecht auf das Alter, wie auf Behinderung und wie auf den Zusammenhang von Alter und Behinderung reagiert. Zum Schluss wird gefragt, ob die sozialrechtlichen Vorkehrungen konsistent sind.

1 Vgl. Welti, Behinderung und Rehabilitation im sozialen Rechtsstaat. Freiheit, Gleichheit und Teilhabe behinderter Menschen. Tübingen 2005; S. 45 ff.; ders. Alter im sozialen Rechtsstaat, BtPrax. 2007, S. 51-55. Igl, Alter und Behinderung im Sozialrecht, in: Berghaus/Bermond/Milz (Hrsg.), Visionen und innovative Konzepte in Zeiten knapper Ressourcen, Kuratorium Deutsche Altershilfe, Köln 2007, S. 12 ff.

2 In der Diskussion um die Einführung eines verbesserten Schutzes bei Pflegebedürftigkeit wurde auch die Festlegung einer Altersgrenze diskutiert, so z.B. Vorschläge der Länder Bayern (BTDrucks. 10/6135) und Rheinland-Pfalz (BR-Drucks. 178/87), s. zur Geschichte die Hinweise bei Igl, Pflegeversicherung, in: von Maydell/Ruland/Becker (Hrsg.), Sozialrechtshandbuch (SRH), 5. Aufl. Baden-Baden 2012, S. 929 ff., 932.

3 S. die Angaben in Pflegestatistik 2009, Statistisches Bundesamt, Wiesbaden 2011. 
Die Sozialrechtswissenschaft befasst sich kaum mit Fragen der möglichen besonderen Schutzbelange von älteren Menschen mit Behinderung. ${ }^{4}$ Die Fragen der Schutzbelange des Alters wurden lange Zeit nur unter dem Aspekt der Sicherung der ökonomischen Subsistenz im Rahmen der monetären Alterssicherung diskutiert. Andere Zusammenhänge, auch die Zusammenhänge von Recht und Alter, wurden bisher eher selten angesprochen. ${ }^{5}$ Allerdings steht zu erwarten, dass sich im Zuge der Altersdiskriminierungsdiskussion auch eine Diskussion jenseits der Fragen der Altersdiskriminierung im Arbeitsleben herausbilden wird. 6

Hingegen werden die rechtlichen Fragen der Schutzbelange von Menschen mit Behinderungen in der Sozialrechtswissenschaft seit einiger Zeit fundiert behandelt. ${ }^{7}$ Mit dem SGB IX hat sich diese Befassung noch verstärkt. Fragen der Eingliederungshilfe für behinderte Menschen nach dem SGB XII sind gerade in jüngerer Zeit Gegenstand rechtlicher, auch rechtspolitischer Befassung. ${ }^{8}$

Vor diesem Hintergrund gesehen befindet sich das zu erörternde Thema „Behinderung und Pflegebedürftigkeit im Alter - sind die sozialrechtlichen Reaktionen konsistent?" in einem Überschneidungsbereich von rechtswissenschaftlicher Artikulation und fast fehlender rechtswissenschaftlicher Befassung.

In dem folgenden Beitrag sollen einige Grundfragen der Herangehensweise an das Thema erörtert werden. Vor allem unter sozialrechtspolitischen Gesichtspunkten soll dies im Abschnitt zu der Frage geschehen, ob das Sozialrecht unterschiedlich auf die Zusammenhänge von „Alter und Behinderung“ und „Behinderung und Alter“ reagiert (Abschnitt II).

Weiter soll einem immer wieder artikulierten Problem der Ausführung der Sozialleistungspraxis nachgegangen werden: Wie sieht es mit der Gewährung von Leistungen der Eingliederungshilfe für ältere Menschen aus? (Abschnitt III). Da zurzeit nicht verlässlich auszumachen ist, ob und in welchem Maße Sozialleistungen die Belange älterer Menschen mit Behinderungen angemessen abdecken, sollen auch Hinweise für den Forschungsbedarf auf diesem Gebiet gegeben werden (Abschnitt IV).

4 Wie Fußn. 1; vgl. auch Igl, Alter und Behinderung im Sozialrecht, in: Hoyer et al. (Hrsg.), Gedächtnisschrift für Jörn Eckert. Baden-Baden, 2008. S. 369-384; ders., Ältere Menschen mit Behinderungen. Realisierung des Teilhabegedankens und Eingliederungshilfe, in: Schütte (Hrsg.), Abschied vom Fürsorgerecht. Von der „Eingliederungshilfe für behinderte Menschen“ zum Recht auf soziale Teilhabe, Berlin 2011, S. 85 ff.

5 Zuletzt Igl/Klie (Hrsg.), Das Recht der älteren Menschen. Baden-Baden 2007; Becker/Roth (Hrsg.), Recht der Älteren, Berlin 2013.

6 S. die umfassende arbeitsrechtliche Analyse von Temming, Altersdiskriminierung im Arbeitsleben. Eine rechtsmethodische Analyse. München, 2008.

7 Welti, Behinderung und Rehabilitation im sozialen Rechtsstaat. Freiheit, Gleichheit und Teilhabe behinderter Menschen, Tübingen 2005.

8 S. den von Schütte herausgegebenen Band und die dortigen Beiträge (wie Fußn. 4). 
II. Unterschiedliche Reaktionen des Sozialrechts auf die Zusammenhänge von „Alter und Behinderung“ und „Behinderung und Alter“ mit Blick auf die Sicherung bei Pflegebedürftigkeit?

\section{Unterschiedliche Lebenssituationen: Der alte Mensch mit Behinderung-} Der behinderte Mensch im Alter

Kehren wir noch einmal zurück zur Unterscheidung von Alter und Behinderung, verstanden als „Alter mit Behinderung“, und Behinderung und Alter verstanden als „Der behinderte Mensch im Alter".

Alter und Behinderung legen eher nahe, dass die Behinderung altersbedingt ist. Pflegebedürftigkeit im Alter ist eine Form der Behinderung im Alter. Damit ist eine bestimmte Wahrnehmung des älteren Menschen im Sinne einer Defizitwahrnehmung 9 verbunden; damit sind auch bestimmte sozialpolitische und sozialrechtliche Reaktionen verbunden. Damit ist vor allem verbunden, dass sich auch in der Bundesrepublik erst spät die Vorstellung durchgesetzt hat, dass Pflegebedürftigkeit ein Phänomen der Behinderung ist. In der sozialrechtlich nicht vorgebildeten Bevölkerung wird man wohl auf Skepsis stoßen, wenn man Pflegebedürftigkeit auch als Behinderung bezeichnet.

Behinderung und Alter hingegen meint, dass die Behinderung schon vor dem Eintritt in die Altersphase gegeben war. Auch damit verbinden sich bestimmte Vorstellungen, werden bestimmte Bilder assoziiert: Die alternde Person mit Down-Syndrom, der ältere körperbehinderte Mensch im Rollstuhl. Dass dieser Personenkreis zusätzlich zur vorhandenen Behinderung im Alter auch noch pflegebedürftig werden kann, wird eher wenig damit assoziiert.

\section{Unterschiedliche sozialrechtliche Reaktionen auf diese unterschiedlichen}

\section{Lebenssituationen?}

\section{a) Fragestellungen}

Die Frage, ob das Sozialrecht mit den unterschiedlichen Lebenssituationen Alter und Behinderung einerseits und Behinderung und Alter andererseits in unterschiedlicher Weise umgeht bzw. umgehen darf, könnte leicht mit dem Verweis auf den Gleichbehandlungsgrundsatz beantwortet werden. Das Sozialrecht mit seinem ausgeprägten Topos der Gewährleistung von gleicher Behandlung in gleichen Situationen wird den behindert gewordenen alten Menschen nicht anders behandeln können als den alt gewor-

9 Hierzu Vierter Altenbericht, BT-Drucks. 14/8822, S. 65 ff. 
denen behinderten Menschen, es sei denn, es ergäben sich gewichtige Unterschiede, die eine Ungleichbehandlung forderten bzw. rechtfertigten.

Das Verständnis der Problematik für eine mögliche unterschiedliche Herangehensweise des Gesetzgebers an die unterschiedlichen Lebenssituationen eröffnet sich wohl erst in einer historischen Betrachtungsweise der sozialen Sicherungssubsysteme für ältere Menschen und für behinderte Menschen. Diese Sicherungssubsysteme haben sich in sehr unterschiedlichen Zusammenhängen und mit sehr unterschiedlichen Zielsetzungen entwickelt. Zudem stehen diese Sicherungssubsysteme in unterschiedlichen Zusammenhängen. Sie haben sich, wie zu sehen sein wird, jedoch in ihren Zielsetzungen aufeinander zu entwickelt.

\section{b) Nichtökonomische Bedarfe im Alter und bei Behinderung}

Nur eine einzige Sozialleistung in unserem so weit gefächerten Sozialleistungssystem nimmt spezifische nichtökonomische altersbedingte Bedarfe wahr und bezieht sich dabei auf das Alter, nämlich die Altenhilfe im Rahmen der Sozialhilfe (§ 71 SGB XII). Die Altenhilfe umfasst auch Leistungen, die für behinderte ältere Menschen in Betracht kommen können, so die Hilfe bei der Beschaffung und zur Erhaltung einer Wohnung, die den Bedürfnissen des alten Menschen entspricht (§ 71 Abs. 2 Nr. 2 SGB XII), die Beratung und Unterstützung bei der Aufnahme in ein Heim (§ 71 Abs. 2 Nr. 3 SGB XII) und bei der Inanspruchnahme altersgerechter Dienste (§ 71 Abs. 2 Nr. 4 SGB XII). Trotzdem ist zu beachten, dass die Behinderung im Alter leistungsrechtlich nicht explizit thematisiert wird.

\section{c) Ökonomischer Bedarf bei Alter und Behinderung}

Spezielle Sicherungen gerade für das Alter sind vor allem die ökonomischen Sicherungen, d.h. die Einkommenssicherung, die von der Rentenversicherung (SGB VI) oder von der Sozialhilfe in Form der Grundsicherung (Viertes Kapitel des SGB XII) besorgt wird. Bei der Grundsicherung, ebenfalls in der Sozialhilfe geregelt, werden behinderungsbedingte Bedarfe nur im Zusammenhang mit der (Geld-)Leistungshöhe der Grundsicherung berücksichtigt ( $§ 42$ Satz 1 Nr. 3 iVm $\S 30$ Abs. 1 Nr. 2 SGB XII).

\section{d) Krankheit im Kontext von Alter und Behinderung}

In der Gesetzlichen Krankenversicherung (SGB V) war für lange Zeit kein besonderer Schutz gerade für ältere Menschen vorgesehen. Dies ist auch heute noch so: Zentraler leistungsauslösender Tatbestand der Gesetzlichen Krankenversicherung ist die Krankheit (vgl. § 11 Abs. 1 Nr. 4, § 27 SGB V). 
Seit der Jahrtausendwende zeichnen sich aber Veränderungen in Richtung auf eine sozialleistungsrechtliche Kenntnisnahme von altersspezifischen Gesundheitsbedarfen ab. So stellt die Erweiterung der medizinischen Rehabilitationsmöglichkeiten eine Leistung dar, die de facto insbesondere für ältere Menschen gelten soll ( $\$ 40$ Abs. 1 und 2 SGB V). ${ }^{10}$ Aber auch die anderen ohne Altersbezug in einer der jüngeren Gesundheitsreformen geschaffenen Leistungen der Palliativversorgung 11 und der Erweiterung der häuslichen Krankenpflege auf das betreute Wohnen und andere Wohnformen ${ }^{12}$ präsentieren sich de facto, d.h. in der statistischen Altersverteilung der Leistungsinanspruchnahme, als Leistungen mit Altersbezug. Die Krankenversicherung nähert sich also - im Gesetzestext nicht explizit ausgesprochen - mit ihren Leistungen den gesundheitlichen Bedürfnissen der älteren Menschen.

\section{e) Pflegebedürftigkeit im Kontext von Alter und Behinderung}

Diese Feststellung gilt wiederum in ganz besonderer Weise für die de-facto-Inanspruchnahme von Leistungen der Sozialen Pflegeversicherung (SGB XI). Dieser Sozialversicherungszweig deckt die speziellen Bedarfe $a b$, die in einer altersspezifischen Behinderungssituation, nämlich der Pflegebedürftigkeit, gegeben sind.

Das Leistungssystem der Sozialen Pflegeversicherung weist bekanntlich ein großes Defizit auf, weil es die Personen mit erheblichem allgemeinem Betreuungsbedarf leistungsrechtlich separiert und damit ein Sonderregime für den - allgemein gesprochen Kreis der Menschen mit Demenz schafft. Immerhin sind in diesem Sonderregime ( $§ 45 \mathrm{a}, 45 \mathrm{~b}$ SGB XI) jetzt auch Personen der sog. Pflegestufe 0 erfasst. Mit der immer noch auf Eis liegenden Erweiterung des Begriffs der Pflegebedürftigkeit könnte hier Abhilfe geschaffen werden. ${ }^{13}$

Erst mit Einführung der Sozialen Pflegeversicherung von 1994 wurde ein Problem des Rehabilitationsgeschehens deutlicher artikuliert: Die Einführung des Vorrangs von Prävention und Rehabilitation vor Pflegeleistungen hat das Bewusstsein für die Rehabilitationsfähigkeit auch des älteren Menschen geöffnet, auch wenn mit dem Vorrangpos-

10 Im Gesetzentwurf zur Gesundheitsreform, BT-Drucks. 16/3100, S. 11, war geplant, Leistungen einzuführen, die gemäß ihrer ursprünglichen Bezeichnung (,geriatrische Rehabilitation“) zum ersten Mal auf eine bestimmte Altersgruppe zugeschnitten sein sollten. Da eine altersgruppenspezifische Rehabilitation jedoch fachlich unangebracht ist, vielmehr die Rehabilitation indikationsspezifisch stattfinden muss, ist in dem zum Gesetz gewordenen Text auf diese Bezeichnung verzichtet worden (vgl. jetzt § 40 SGB V). S. zu dieser Thematik Fuchs, Rechtliche Rahmenbedingungen für die geriatrische Rehabilitation. Was hat sich durch die Gesundheitsreform geändert? Soziale Sicherheit 2007, S. 169 ff.

$11 \S 37 \mathrm{~b}$ SGB V, eingeführt durch Art. 1 Nr. 23 des Gesetzes v. 26.3.2007, BGBl. I 2007, S. 378 mit Wirkung vom 1.4.2007.

$12 \S 37$ SGB V, geändert durch Art. 1 Nr. 22 Buchst. a des Gesetzes v. 26.3.2007, BGB1. I 2007, S. 378 mit Wirkung vom 1.4.2007.

13 S. hierzu Wilcken, Pflegebedürftigkeit und Behinderung im Recht der Rehabilitation und Teilhabe und im Recht der Pflege, Berlin 2011 (zugl. Diss. Kiel 2011), S. 300 ff. 
tulat „nur“ die medizinische Rehabilitation gemeint ist (vgl. § 5 SGB XI). Dieses Postulat des Vorrangs von Prävention und medizinischer Rehabilitation blieb mehr als zehn Jahre nach Einführung der Pflegeversicherung meist mehr Ausdruck eines Wunsches als Umsetzung in die Realität. In einer der jüngeren Gesundheitsreformen ${ }^{14}$ musste der Gesetzgeber das gesetzliche Instrumentarium zur Realisierung dieses Rehabilitationsanliegens nach mehreren Versuchen noch einmal schärfen, damit die Sozialleistungsträger den gesetzlichen Auftrag als verbindlichen Auftrag begreifen und damit nicht nur der Vorrang der Prävention und Rehabilitation, sondern auch der Vorrang des Gesetzes, d.h. die Beachtung des Gesetzes durch die Sozialleistungsträger, hier endlich Realität wird.

Vielleicht haben die Schwierigkeiten bei der Realisierung des Postulates „Rehabilitation vor Pflege" auch damit zu tun, dass es im deutschen Rehabilitationswesen lange Zeit unüblich war, ältere Menschen zu rehabilitieren, ohne dass es um die Wiedereingliederung in den Arbeitsmarkt ging. Dazu gleich noch einmal:

\section{f) Behinderung im Alter: Teilhabe und Rehabilitation}

Der sozialrechtliche Schutz behinderter Menschen hat mit dem SGB IX explizit die Zielsetzung der Förderung der gleichberechtigten Teilhabe am Leben in der Gesellschaft erhalten (§ 1 Satz 1 SGB IX). Dieser Zielsetzung dienen die Leistungen zur Teilhabe ( $\$ 4$ Abs. 1 SGB IX). Diese moderne Rahmensetzung für den sozialen Schutz behinderter Menschen lässt leicht in Vergessenheit geraten, dass das deutsche Sozialleistungssystem seine Teilhabebemühungen lange Zeit vornehmlich auf die (Wieder)Erlangung von Erwerbsfähigkeit konzentriert hat. Dem alten und behinderten Mensch hat man trotz eines ausgebauten Rehabilitationswesen keine besonderen Rehabilitationsbemühungen angedeihen lassen. Der alte Mensch hat als nicht mehr rehabilitationsfähig gegolten. Was eine solche Wahrnehmung in der Sache ausmacht, wird erst dann besonders deutlich, wenn man dies in die heutige Terminologie übersetzt und nicht sagt, der alte Mensch ist nicht mehr rehabilitationsfähig, sondern, der alte Mensch ist nicht mehr teilhabefähig. Freilich gab es auch schon vor dem SGB IX von 2001 Rehabilitationsleistungen, häufig betitelt als Leistungen zur sozialen Rehabilitation, denen die in $\S 4$ Abs. 1 Nr. 4 SGB IX formulierte Zielsetzung der gesellschaftlichen Teilhabe innewohnte, so in der Eingliederungshilfe des Sozialhilferechts.

Die Eingliederungshilfe des Sozialhilferechts verfügte schon im Bundessozialhilfegesetz (BSHG) über die breiteste Leistungsspreizung für behinderte Personen (vgl. $\S \S 53,54$ SGB XII) und wurde deshalb der allgemeinen Teilhabezielsetzung am ehesten gerecht. Das höhere Lebensalter war, abgesehen von den schulischen Leistungen, ge-

14 Hierzu Igl, Das Gesetz zur strukturellen Weiterentwicklung der Pflegeversicherung, NJW 2008, S. $2214 \mathrm{ff}$. 
setzlich nicht als Ausschlusskriterium von bestimmten Leistungen vorgesehen. ${ }^{15}$ Aber ein Blick in die Eingliederungshilfe-Verordnung ${ }^{16}$ zeigt, dass sich das Leistungsspektrum auf die schulische und berufliche Ausbildung und die Eingliederung in das Arbeitsleben konzentriert. Spezielle Teilhabeleistungen für ältere Menschen sieht die Eingliederungshilfe-Verordnung nicht vor.

\section{g) Die Wirkungen der Wahrnehmung von Alter, Behinderung und \\ Pflegebedürftigkeit in den einzelnen Sozialleistungssystemen}

Das Sozialleistungssystem reagiert erst in jüngerer Zeit auf die besonderen Belange im Zusammenhang von Alter und Behinderung, allerdings nicht auf eine Weise, in der sich der Zusammenhang von Alter und Behinderung leistungsrechtlich explizit abbilden würde. Die Leistungsbereiche, in denen gerade der Zusammenhang von Alter und Behinderung eine Rolle spielt, nennen diesen Zusammenhang nicht. Dies gilt für die Leistungen bei Pflegebedürftigkeit. Diese Leistungen kommen statistisch ganz überwiegend hochaltrigen Personen zugute. Der Zusammenhang von Alter und Behinderung versteckt sich, so gesehen, leistungsrechtlich hinter dem Begriff der Pflegebedürftigkeit.

Die Wahrnehmung von älteren Menschen als medizinisch rehabilitationsfähigen Personen ist ebenfalls der Pflegeversicherung zu verdanken. Der gesetzlich angeordnete Vorrang von Prävention und medizinischer Rehabilitation vor Pflege hat hierfür das Verständnis eröffnet.

Auch in der Gesetzlichen Krankenversicherung sind in der letzten Dekade Leistungen aufgenommen worden, die - wiederum ohne es zu benennen - vor allem älteren Menschen zugutekommen sollen.

Der Zusammenhang von Alter und Behinderung bildet sich somit sozialleistungsrechtlich in drei Dimensionen ab: in der Dimension des Schutzes bei Pflegebedürftigkeit, in der Dimension der medizinischen Rehabilitationsfähigkeit und in der Dimension spezieller Gesundheitsleistungen. Allen drei Dimensionen liegt eine Form der Behinderung zugrunde, und allen drei Dimensionen ist gemeinsam, dass die Leistungen de facto, das heißt in der statistischen Verteilung, für Menschen höheren Alters besonders einschlägig sind.

Diese Reaktionen auf den Zusammenhang von Alter und Behinderung zeigen zweierlei: Erstens steht im Vordergrund des Sozialleistungsrechts der ältere Mensch, der im Alter behindert in Form der Pflegebedürftigkeit geworden ist, nicht der behinderte Mensch, der älter geworden ist. Zweitens wird das Alter nicht zum leistungsauslösenden Kriterium erhoben, was im Übrigen dem Gleichbehandlungsgrundsatz sehr entspricht.

15 Das wird auch in der Rechtsprechung immer wieder betont, s. zuletzt den Beschluss des LSG Baden-Württemberg vom 19.03.2012, Az.: L 2 SO 72/12 ER-B, Rdnr. 10, zitiert nach juris.de. S. auch BVerwG v. 21.12.2005, Az.: 5 C 26/04, Rdnr. 13, zitiert nach juris.de = NVwZ-RR 2006, 406.

16 Verordnung nach $\S 60$ SGB XII (Eingliederungshilfe-Verordnung). 
Damit bleiben vor allem die Leistungen der Eingliederungshilfe des SGB XII für jene Personen bestimmt, die als behinderte Menschen alt geworden sind. Im SGB XII sind das vor allem die Leistungen, die nicht eine schulische oder berufliche Rehabilitation zum Gegenstand haben.

Der Ausgangspunkt für das Sozialleistungsrecht ist für den behinderten Menschen im Alter ein anderer als für den im Alter behindert, d.h. pflegebedürftig gewordenen Menschen. Der behinderte Mensch erhält, bevor er älter wird, Leistungen, die ihm die Teilhabe in den verschiedenen Lebensbereichen sichern sollen (vgl. § 4 Abs. 1 SGB IX). Diese Teilhabeleistungen, die im Lebensabschnitt des Erwerbstätigenalters vor allem Leistungen zur Teilhabe am Arbeitsleben sind, stellen in diesem Lebensabschnitt das Zentrum des Rehabilitationsgeschehens dar. Alle anderen Leistungen zur Teilhabe gruppieren sich um die Teilhabe am Arbeitsleben, sie stehen aber nicht im Zentrum. Wenn dieses Zentrum bei Erreichen der Rentenaltersgrenze wegfällt, kommt dann den anderen Teilhabeleistungen mehr Gewicht zu.

\section{Sozialleistungspraxis bei älteren behinderten Menschen}

\section{Fragestellungen}

Im Folgenden soll nun einem Problem nachgegangen werden, das mit einem mancherorts ausgesprochenem Unbehagen zu tun hat, nämlich der Frage, ob gerade bei behinderten Menschen ab einer gewissen Schwelle des höheren Alters Leistungen der Eingliederungshilfe nicht mehr gewährt werden, und ob diese Personen ,,in die Pflegeversicherung abgeschoben“ werden. Das würde, etwas verkürzt gesagt, bedeuten, dass diesem Personenkreis Leistungen zur Teilhabe am Leben in der Gemeinschaft aus Altersgründen versagt würden. ${ }^{17}$ Wie es dann mit dem Personenkreis aussieht, der erst im Alter behindert, d.h. pflegebedürftig geworden ist, und der dann ebenfalls zusätzlich auf Teilhabeleistungen angewiesen ist, wäre dann die andere Seite der Betrachtung der Realisierung des Teilhabegedankens bei älteren Menschen mit Behinderung.

Bei der Erörterung dieser Fragen kann man es möglicherweise auch mit einer spezifischen Diskriminierungsproblematik gegenüber älteren Menschen beim Sozialleistungsbezug zu tun haben. Deshalb zunächst einige Worte zu diesem Thema.

17 S. hierzu auch den Beitrag von Welti, Alter im sozialen Rechtsstaat, BtPrax. 2007, S. 51 ff. 


\section{Allgemeine Probleme der Feststellung einer altersspezifischen Leistungspraxis} bei Sozialleistungen

In verschiedenen Zusammenhängen wird eine altersspezifische Leistungspraxis bei Sozialleistungen gerügt. ${ }^{18} \mathrm{Zu}$ unterscheiden ist dabei zwischen einer altersspezifischen Leistungszuweisung (Leistungszugang) und einer altersspezifischen Art und Weise der Leistungserbringung.

In die erste Kategorie fallen dabei die rationierte Zuteilung bzw. die Nichtgewährung von bestimmten Sozialleistungen an ältere Menschen. Die Debatte hierüber kann an dieser Stelle nicht aufgenommen werden. Sie betrifft vor allem bestimmte Gesundheitssozialleistungen für Menschen ab einem definierten höheren Lebensalter. In diese erste Kategorie (Leistungszugang) fällt auch eine spezifische Praxis von Sozialleistungsträgern, Leistungen für ältere Menschen nicht mehr zu gewähren, obwohl die Leistungsvoraussetzungen hierfür noch gegeben sind. Dies gilt für die Nicht- und Nichtweitergewährung von Leistungen der Eingliederungshilfe für ältere Menschen auf dem Gebiet der Sozialhilfe. Diesem Thema soll im Folgenden nachgegangen werden.

Die zweite Kategorie einer altersspezifischen Leistungspraxis betrifft die Art und Weise der Leistungserbringung, z.B. den Umfang und die Intensität einer medizinischen Behandlung bei einem älteren Menschen im Vergleich zu einem Menschen im jüngeren Alter. Im Zusammenhang dieser Kategorie wird häufig eine Bewertung vorgebracht im Sinne einer besseren/schlechteren Behandlung. Allerdings wird manchmal nicht gesehen, dass eine andere Art und Weise der Behandlung im Alter unter Umständen fachlich geboten sein kann. So sieht beispielsweise die Psychiatrie-Personalverordnung ${ }^{19}$ in der Personalbemessung in der Gerontopsychiatrie im Vergleich zur allgemeinen Psychiatrie geringere Minutenwerte für den Einsatz des ärztlichen Personals vor (§ 5 Abs. 1 PsychPV). Daraus kann aber nicht gefolgert werden, dass der Kreis der gerontopsychiatrischen Patientinnen und Patienten medizinisch schlechter versorgt wird als derjenige der allgemeinen Psychiatrie. Vielmehr ist, spezifisch an den Therapiebedarfen der gerontopsychiatrischen Patientinnen und Patienten orientiert, sogar ein verstärkter Einsatz von Krankenpflegepersonal und Bewegungstherapeuten, Krankengymnasten und Physiotherapeuten vorgesehen ( 5 Abs. 1 Psych-PV).

18 Die Angaben oder Hinweise sind jedoch zum Teil wenig verlässlich. An umfassenden und belastbaren repräsentativen Untersuchungen fehlt es bislang.

19 Verordnung über Maßstäbe und Grundsätze für den Personalbedarf in der stationären Psychiatrie (Psychiatrie-Personalverordnung - Psych-PV). 


\section{Zur Problematik der Leistungspraxis der Eingliederungshilfe für ältere}

\section{behinderte Menschen}

\section{a) Fragestellungen}

Ein besonderes Problem stellt die teilweise vermutete Praxis der Sozialleistungsträger bei der Vergabe von bestimmten Leistungen der Eingliederungshilfe für ältere Menschen dar, dies auch an der Schnittstelle zu den Leistungen der Pflegeversicherung. Hier wird teilweise - allerdings nicht belastbar - vorgebracht, dass sich gewisse Praktiken der Leistungsverlagerung bzw. der Leistungsversagung vor allem im Altersbereich zwischen 60 und 65 Jahren ergeben haben.

Den weiteren Ausführungen ist vorwegzuschicken, dass in der Bundesrepublik keine repräsentative oder gar flächendeckende Untersuchung zur Leistungspraxis der Sozialhilfeträger existiert. Verwaltungsinterne Anweisungen, Verwaltungsvorschriften in den verschiedenen rechtlichen Ausprägungen und andere untergesetzliche Vorschriften sind oft nicht publiziert oder zugänglich. Die Sozialhilferichtlinien der Länder sind nur in wenigen Fällen im Internet recherchierbar. In den großen juristischen Datenbanken sind sie - soweit ersichtlich - nicht enthalten.

Hier ist bereits Forschungsbedarf angemahnt worden. ${ }^{20}$ Bis jetzt liegen allerdings keine einschlägigen Untersuchungen vor. Der Sechste Altenbericht ${ }^{21}$, vorgelegt im Juni 2010, enthält ein Kapitel zu Altersgrenzen bei Sozialleistungen im Bereich Gesundheit, Pflege und Eingliederung. Leider hat es die Altenberichtskommission versäumt, hier einschlägige Gutachten einzuholen, damit endlich mit belastbaren Daten gearbeitet werden kann. Die Bundesregierung ist in ihrer Stellungnahme zum 6. Altenbericht nicht auf diese Problematik eingegangen. ${ }^{22}$

\section{b) Versuch einer Annäherung an die Problematik}

Man kann die geschilderte vermutete Leistungspraxis der Eingliederungshilfe für ältere behinderte Menschen unter dem Aspekt der Rationierung von bestimmten Sozialleistungen betrachten. Die Diskussion um die Rationierung bestimmter Leistungen aus Altersgründen findet in der Bundesrepublik mittlerweile breit statt. Sie konzentriert und beschränkt sich allerdings auf die Thematik der Rationierung von Gesundheitsleistungen. ${ }^{23}$ Die dort gebräuchliche Unterscheidung von impliziter und expliziter Rationie-

$20 \mathrm{Igl}$, in: Schütte (Fußn. 4), S. 95 f.

21 BT-Drucks. 17/3815, Sechster Bericht zur Lage der älteren Generation in der Bundesrepublik Deutschland. Altersbilder in der Gesellschaft und Stellungnahme der Bundesregierung, S. 206 f.

22 Igl, in: Schütte (Fußn. 4), S. I ff.

23 So auch die entsprechende Expertise für den 6. Altenbericht (Fußn. 21) von Remmers, Rationierung und Altersdiskriminierung, in: Berner/Rossow/Schwitzer (Hrsg.), Altersbilder in der Wirtschaft, im 
rung ${ }^{24}$ und die Unterscheidung nach den Wirkungsebenen (Mikro-, Meso- und Makroebene) ${ }^{25}$ kann für die hier vorliegende Problematik zumindest begrenzt weiter helfen.

Versteht man unter Rationierung die Zuteilung begrenzt vorhandener Güter nach bestimmten Kriterien, dann wären die begrenzt vorhandenen Güter hier bestimmte Leistungen der Eingliederungshilfe, die dem Buchstaben und Sinn des Gesetzes entsprechend nur gemäß den gesetzlich beschriebenen Kriterien, nicht aber gemäß implizit angebrachten Kriterien wie Alter und/oder Pflegebedürftigkeit zugeteilt werden dürften. Diese implizite Rationierung würde dann auf der Mikroebene der Sozialleistungsgewährung, also im Verhältnis zwischen Sozialhilfeträger und Sozialhilfeberechtigtem, stattfinden. Wenn Sozialhilfeträger ein solches Verhalten gesteuert, z.B. durch entsprechende Richtlinien veranlasst, pflegen, würde die implizite Rationierung auf der Mesoebene organisiert werden. Dass eine solche implizite Rationierung in Widerspruch zum Gesetz steht, bedarf keiner näheren Erläuterung.

Die Besonderheit einer solchen Rationierung kann aber darin gesehen werden, dass die Sozialhilfeträger aus ihrer Sicht die betroffenen Personen nicht ohne Leistung lassen, sondern diese im Leistungssystem der Sozialen Pflegeversicherung als gut aufgehoben und versorgt betrachten. Solche Erwägungen - auch hier kann man nur aus eigenen Wahrnehmungen berichten - sind Sozialhilfeträgern nicht fremd. Schon bei Einführung der Pflegeversicherung ist bekannt geworden, dass es viele Bemühungen seitens der Sozialhilfeträger gegeben hat, behinderte Menschen in die Pflegeversicherung ,abzuschieben“. Vor diesem Hintergrund erlangt § 43a SGB XI - Reduzierung der Pflegeleistungen für Pflegebedürftige in vollstationären Einrichtungen der Hilfe für behinderte Menschen - eine besondere Konnotation.

Bei meinen eigenen statistischen Analysen zum Thema der Leistungspraxis der Eingliederungshilfe ${ }^{26}$ konnte ich immerhin zwei Ergebnisse festhalten:

- Der einwohnerproportionale Anteil der Empfänger von Leistungen der Eingliederungshilfe im Laufe des Berichtsjahres 2008 variiert zwischen den Bundesländern erheblich. ${ }^{27}$

- Mit zunehmendem Alter der Leistungsempfänger steigen die Anteile der nicht altersspezifischen Leistungen der Eingliederungshilfe von Alterskohorte zu Alterskohorte an. Lediglich die Hilfen zur Förderung der Verständigung mit der Umwelt bleiben anteilig fast auf gleicher Höhe, während der Anteil der anderen Leistungen zur Teilhabe am Leben in der Gemeinschaft fast um die Hälfte abnimmt. Im Verhältnis zu allen Beziehern von Leistungen der Eingliederungshilfe stellt

Gesundheitswesen und in der pflegerischen Versorgung. Expertisen zum Sechsten Altenbericht der Bundesregierung, Band 2, Wiesbaden 2012, S. 339-368.

$24 \mathrm{Igl}$, in: Schütte (Fußn. 4), S. 343 f.

25 Igl, in: Schütte (Fußn. 4), S. 345 f.

26 Igl, in: Schütte (Fußn. 4), S. 92 ff.

27 Mittelwert Bundesrepublik: 8,7; höchster Wert in Mecklenburg-Vorpommern mit 13,0; niedrigste Werte in Baden-Württemberg $(6,0)$ und Bremen $(5,9)$, jeweils bezogen auf 1000 Einwohner; Statistisches Bundesamt, 2010, Tabelle B. 6.2. 
sich der Anteil der nicht altersspezifischen Leistungen bei den Hilfen zum selbstbestimmten Wohnen und bei den Leistungen zur Teilhabe am Leben in der Gemeinschaft in den jeweiligen Alterskohorten als ansteigend dar.

Mit diesen Ergebnissen ist sicher noch nicht viel gewonnen. Sie zeigen aber, dass ein pauschalierender Rationierungsvorwurf gegenwärtig mangels belastbaren Datenmaterials spekulativ bleibt. Nochmals ist also Forschungsbedarf anzumelden.

\section{Ergebnisse und Schlussfolgerungen}

Die Realisierung des Teilhabegedankens für ältere Menschen mit Behinderung hängt nicht nur von der sozialleistungsrechtlichen Situation, sondern auch von der Praxis der Sozialleistungsgewährung ab.

\section{Sozialleistungsrecht}

Das Sozialleistungsrecht bildet die besonderen Schutzbelange im Zusammenhang von Alter und Behinderung nicht explizit und nicht in allgemeiner Weise ab. Der $\mathrm{Zu}$ sammenhang von Alter und Behinderung bildet sich sozialleistungsrechtlich aber in drei speziellen Dimensionen ab: in der Dimension des Schutzes bei Pflegebedürftigkeit, in der Dimension der medizinischen Rehabilitationsfähigkeit und in der Dimension spezieller Gesundheitsleistungen. Allen drei Dimensionen liegt eine Form der Behinderung zugrunde, und allen drei Dimensionen ist gemeinsam, dass die Leistungen de facto, das heißt in der statistischen Verteilung, für Angehörige der höheren Altersgruppen besonders einschlägig sind. Das Sozialleistungsrecht unterscheidet auch nicht danach, ob ein älterer Mensch im Alter behindert wird, oder ob ein behinderter Mensch älter wird.

Die Frage, ob die sozialleistungsrechtlichen Vorkehrungen in besonderer Weise den Belangen älterer Menschen mit Behinderungen, auch in Form von Pflegebedürftigkeit, Rechnung tragen sollten, muss dann mit Nein beantwortet werden, wenn mit den vorhandenen Leistungen bereits diesen Belangen Rechnung getragen wird. Wenn dies der Fall ist, bedarf es keiner eigenen Kategorie von Leistungen für ältere Menschen mit Behinderungen. Alleine die Tatsache, dass bestimmte Sozialleistungen statistisch von Angehörigen der älteren Generation in höherem Maß als von Angehörigen jüngerer Generationen in Anspruch genommen werden, spricht noch nicht dafür, dass solche besonderen Kategorien von Leistungen explizit, d.h. nur für ältere Menschen mit Behinderungen, geschaffen werden sollten. Dies hätte für andere, situativ gleich betroffene Personen Nachteile, denn jede gesetzlich angebrachte Altersgrenze wirkt für Personen jenseits des anspruchsbegründenden Alters leistungsausschließend. Solange gewährleistet ist, dass das vorhandene Sozialleistungsspektrum die Belange älterer Menschen mit 
Behinderungen abdeckt, ist daher von besonderen Leistungen für diesen Personenkreis abzusehen. Das setzt aber voraus, dass in der Leistungspraxis das Leistungsspektrum ausgeschöpft wird.

\section{Sozialleistungsrechtliche Praxis}

In der sozialleistungsrechtlichen Praxis werden Andeutungen gemacht und Vermutungen geäußert, dass die Gewährung von Leistungen der Eingliederungshilfe des SGB XII an ältere Menschen mit Behinderungen eingeschränkt wird, u.a. auch mit dem Hinweis auf mögliche Leistungen der Pflegeversicherung (SGB XI). Anhand des vorliegenden statistischen Materials sind diese Annahmen nicht belegbar. Die vorliegenden Daten deuten sogar darauf hin, dass ältere Menschen mit Behinderungen bestimmte Teilhabeleistungen der Eingliederungshilfe mit höherem Anteil erhalten als jüngere Menschen mit Behinderungen. Sozialwissenschaftliche fundierte und belastbare Daten und Erkenntnisse liegen jedoch nicht vor.

\section{Forschungsbedarf}

Angesichts der unbefriedigenden Erkenntnisse über die Praxis der Gewährung von Teilhabeleistungen für ältere Menschen mit Behinderungen ist es angezeigt, hier entsprechende Untersuchungen vorzunehmen. Diese Untersuchungen sollten in zwei Richtungen gehen:

- Feststellung des Anteils der Inanspruchnahme der einzelnen Hilfen im Rahmen der Eingliederungshilfe nach dem SGB XII durch ältere Menschen mit Behinderungen im Vergleich zu jüngeren Menschen mit Behinderung (Bundes-, Länderund Sozialhilfeträgervergleich);

- Untersuchung der Frage nach einer Praxis von Sozialhilfeträgern, ob ab einer bestimmten Altersgrenze bestimmte Leistungen der Eingliederungshilfe versagt und ggf. auch durch Leistungen der Pflegeversicherung abgelöst werden.

Diese Untersuchungen könnten auch unter dem Gesichtspunkt durchgeführt werden, ob die Teilhabebelange älterer Menschen mit Behinderungen mit dem vorhandenen Sozialleistungsspektrum angemessen abgedeckt sind und ob ggf. ein Bedarf an weiteren Leistungen oder an Veränderungen bzw. Präzisierungen vorhandener Leistungen besteht. $^{28}$

28 Für seelisch behinderte Kinder und Jugendliche ist ein solcher besonderer Schutz bereits realisiert, vgl. § 35a SGB VIII. Hierzu Greß/Rixen/Wasem, Eingliederungshilfe für seelisch behinderte Kinder und Jugendliche: Abgrenzungsprobleme und Reformszenarien, Vierteljahresschrift für Sozialrecht 2009, S. $43 \mathrm{ff}$. 
Mit diesen Untersuchungen könnte auch ein Beitrag zu der Forderung der 85. ASMK $^{29}$ nach einer personenzentrierten teilhabeorientierten Weiterentwicklung des Eingliederungshilfesystems geleistet werden.

\section{Zur Eingangsfrage}

Auf die in der Überschrift gestellte Frage ,Sind die sozialrechtlichen Reaktionen auf Behinderung und Pflegebedürftigkeit im Alter konsistent?“ kann mit zwei Sätzen im Konjunktiv geantwortet werden:

Die sozialrechtlichen Reaktionen wären konsistent,

- wenn in der Praxis der Eingliederungshilfe das vorhandene Leistungsspektrum auch für ältere behinderte und pflegebedürftige Menschen ausgeschöpft würde, und

- wenn der Begriff der Pflegebedürftigkeit so gefasst und gesetzlich implementiert würde, dass die Teilhabe auch der Menschen mit allgemeinem Betreuungsbedarf gesichert ist.

Der Antwort auf die in der Überschrift gestellte Frage ist hinzuzufügen, dass Pflegebedürftigkeit als Ausdruck einer Behinderung zu sehen ist. Damit würden die Leitprinzipien des SGB IX auch für pflegebedürftige Menschen gelten. Damit würde auch die Selbstverständlichkeit der Geltung dieser Leitprinzipien bei der Aufgabenwahrnehmung der Sozialhilfeträger in Richtung auf behinderte ältere Menschen unterstrichen werden, die heute noch als pflegebedürftig, nicht aber als behindert angesehen werden.

29 85. Arbeits- und Sozialministerkonferenz 2008, Weiterentwicklung der Eingliederungshilfe für Menschen mit Behinderungen. Hamburg, 13./14. November 2008. 


\title{
Behindertenrecht und Behindertenpolitik in der Kommune - ein Blick in die Rechtsprechung
}

\author{
Stephan Rittweger
}

I. Einleitung

II. Entwicklungen in der neueren Rechtsprechung

1. Kein Auto zulasten der Sozialhilfe

2. § 71 SGB XII: Kulturelle Teilhabe, kein Anspruch auf Theaterbesuch

3. Kein Sport-Rollstuhl - Krankenversicherung

4. Inklusionsziel Bildung

5. Kein Cialis zulasten der Gesetzlichen Krankenversicherung

III. Zusammenfassung und Ausblick

\section{Einleitung}

Die Gerichte haben als Dritte Gewalt im Staate die klassische Aufgabe, die anderen Staatsgewalten zu kontrollieren. Die Sozialgerichte haben den speziellen Auftrag, die Sozialleistungsträger zu überprüfen und sie immer wieder anzuhalten, dass jedem Berechtigten die ihm zustehenden Sozialleistungen in zeitgemäßer Weise, umfassend und zügig zukommen vgl. § 17 SGB I. Dabei hat das Bundesverfassungsgericht die Sozialgerichte schon manches Mal daran erinnert, dass sie sich schützend und fördernd vor die Rechtsgüter der Betroffenen stellen sollen. Diese Aufgabenstellung erfordert, Gerichtsverfahren einem zeitnahen Ende durch gütliche Einigung zuzuführen. Klagen über mehrere Instanzen und Jahre hinweg schaden oft mehr als sie den Betroffenen nutzen. Die weit überwiegende Zahl der Verfahren vor den Sozialgerichten endet deshalb unstreitig, Anerkenntnisse und Vergleiche beenden weitaus mehr Verfahren als Urteile und Beschlüsse. Das ist der Grund, warum ein Überblick über die sozialgerichtliche Rechtsprechung keineswegs vollständig abbildet, wie Sozialbehörden und Sozialgerichte zur Inklusion stehen.

Die Aufgaben der sozialgerichtlichen Rechtsprechung erschöpfen sich aber nicht in der Schaffung von Rechtsfrieden im Einzelfall. An vielen Stellen des Sozialgesetzbuches hat der Gesetzgeber unbestimmte Rechtsbegriffe verwendet, ja verwenden müssen. Deren Inhalte zu bestimmen ist eine weitere bedeutsame Aufgabe der Gerichte. Ihre 